Article

\title{
Flavonoids and a New Polyacetylene from Bidens parviflora Willd.
}

\author{
Yu-Lan $\mathrm{Li}^{1,2}$, Jun $\mathrm{Li}^{2}$, Nai-Li Wang ${ }^{1, *}$ and Xin-Sheng Yao ${ }^{1, *}$ \\ ${ }^{1}$ Department of Natural Products Chemistry, Shenyang Pharmaceutical University, 103 Wenhua \\ Road, Shenhe District, Shenyang, Liaoning 110016, P.R. China; E-mail: liyulan@szda.gov.cn(Yu- \\ Lan Li) \\ 2 Shenzhen Institute for Drug Control, 1024, Beihuan Road, Futian District, Shenzhen, Guangdong \\ 518029, P.R. China; E-mail: lijun@szda.gov.cn (Jun Li)
}
* Authors to whom correspondence should be addressed; E-mails: wangnl@sz.tsinghua.edu.cn (Nai-Li Wang); yaoxinsheng@vip.tom.com (Xin-Sheng Yao); Tel: 86-20-85225849; Fax: 86-20-85221559.

Received: 20 July 2008; in revised form: 19 August 2008 / Accepted: 26 August 2008 / Published: 28 August 2008

\begin{abstract}
Fifteen flavonoids, 1-7 and 9-16, and a polyacetylene, 8, were isolated from the ethanol extract of the dried whole plant of Bidens parviflora Willd. by various chromatographic techniques. Their structures have been elucidated on the basis of spectroscopic analyses and chemical studies. Compound $\mathbf{8}$ is new and was identified as 3$(R), 8(E)$-decene-4,6-diyne-1,3,10-triol. All the flavonoid compounds were isolated for the first time from this plant species.
\end{abstract}

Keywords: Bidens parviflora Willd,; Polyacetylene; Flavonoids.

\section{Introduction}

The plant Bidens parviflora Willd. is used in Chinese folk medicine as an antipyretic, antiinflammatory and antirheumatic [1, 2]. Flavones [3], flavonones [4], flavonoid glycosides [3, 5], flavonol glycosides [6,7], chalcones [8, 9], aurones [7, 8], sterols [10], polyacetylene glucosides [4, 9] and monoterpenes $[4,10]$ have all been previously reported in this species. In our previous studies, sucrose esters [11], phenolic acids [12], polyacetylene glucosides [13], monoterpene glycosides [14], 
neolignan glucosides [15], phenolic glucosides [16] and caffeoylquinic acid derivatives [17] were isolated from this plant. As a part of an ongoing research program, this paper describes the isolation and structural determination of a new ployacetylene, 8, and 15 known flavonoids from $B$. parviflora Willd. (Figure 1).

Figure 1. The structures of compounds 1-16.<smiles>[R20]c1cc(O)c2c(=O)c([R])c(-c3ccc([R20])c([R])c3)oc2c1</smiles>

1: $\mathrm{R}_{1}=-\alpha$-D-apiofuranosyl $(1 \rightarrow 6)-\beta$-D-glucose, $\mathrm{R}_{2}=\mathrm{CH}_{3}, \mathrm{R}_{3}=\mathrm{R}_{4}=\mathrm{H}$

2: $\mathrm{R}_{1}=-\beta$-D-neohesperidose, $\mathrm{R}_{2}=\mathrm{R}_{4}=\mathrm{H}, \mathrm{R}_{3}=\mathrm{OH}$

3: $\mathrm{R}_{1}=\mathrm{R}_{2}=\mathrm{H}, \mathrm{R}_{3}=\mathrm{OH}, \mathrm{R}_{4}=-\mathrm{O}-\beta$-D-rutinose

4: $\mathrm{R}_{1}=-\beta$-D-glucose, $\mathrm{R}_{2}=\mathrm{R}_{4}=\mathrm{H}, \mathrm{R}_{3}=\mathrm{OH}$

5: $\mathrm{R}_{1}=-\beta$-D-glucose, $\mathrm{R}_{2}=\mathrm{CH}_{3}, \mathrm{R}_{3}=\mathrm{R}_{4}=\mathrm{H}$

6: $\mathrm{R}_{1}=\mathrm{R}_{2}=\mathrm{H}, \mathrm{R}_{3}=\mathrm{OH}, \mathrm{R}_{4}=-\mathrm{O}-\alpha$-L-rhamnose

9: $\mathrm{R}_{1}=\mathrm{R}_{2}=\mathrm{H}, \mathrm{R}_{3}=\mathrm{OH}, \mathrm{R}_{4}=-\mathrm{O}-\beta$-D-glucose

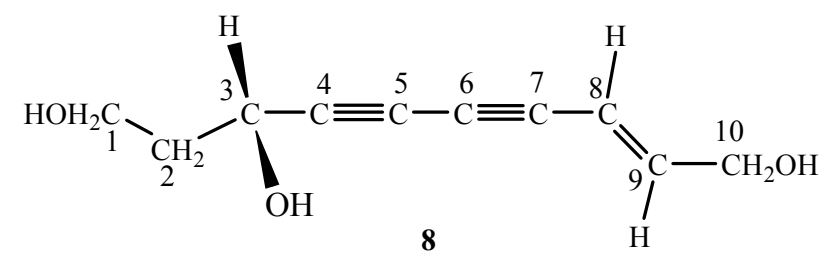<smiles>[R]c1cc(-c2oc3cc(O)cc([R])c3c(=O)c2[R])ccc1O</smiles>

12: $\mathrm{R}_{1}=\mathrm{R}_{3}=\mathrm{OH}, \mathrm{R}_{2}=\mathrm{H}$ 13: $\mathrm{R}_{1}=\mathrm{R}_{2}=\mathrm{OH}, \mathrm{R}_{3}=\mathrm{H}$ 14: $\mathrm{R}_{1}=\mathrm{R}_{2}=\mathrm{R}_{3}=\mathrm{OH}$

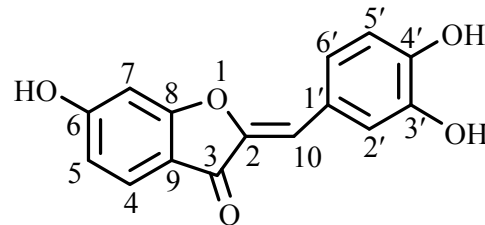

15

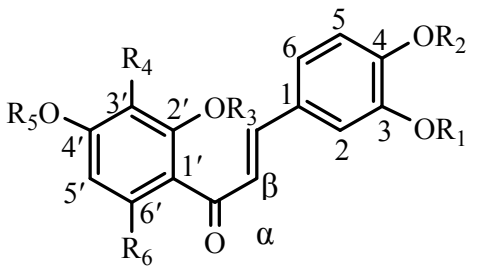

7: $\mathrm{R}_{1}=\mathrm{R}_{3}=\mathrm{R}_{6}=\mathrm{H}, \mathrm{R}_{2}=\mathrm{CH}_{3}, \mathrm{R}_{4}=\mathrm{OH}, \mathrm{R}_{5}=-\beta$-D-glucose 11: $\mathrm{R}_{1}=\mathrm{R}_{2}=\mathrm{R}_{5}=\mathrm{CH}_{3}, \mathrm{R}_{3}=\mathrm{R}_{4}=\mathrm{H}, \mathrm{R}_{6}=\mathrm{OCH}_{3}$ 16: $R_{1}=R_{2}=R_{3}=R_{4}=R_{5}=R_{6}=H$

\section{Results and Discussion}

Compounds 1-7 and 9-16 were identified as acacetin $7-O-(\alpha-\mathrm{D}$-apio-furanosyl $)(1 \rightarrow 6)-\beta$-Dglucoside (1), luteolin 7-O- $\beta$-D-neohesperidoside (2), quercetin 3- $O$ - $\beta$-D-rutinoside (3), luteolin 7- $O$ $\beta$-D-glucoside (4), acacetin 7-O- $\beta$-D-glucoside (5), quercitrin (6), 4-methoxyl-3,2',3'-trihydroxychalcone 4'-O- $\beta$-D-glucoside (7), quercetin 3- $O$ - $\beta$-D-glucoside (9), taxifolin (10), $2^{\prime}$-hydroxy-3,4,4',6'tetramethoxychalcone (11), kaempferol (12), luteolin (13), quercetin (14), sulfuretin (15) and 3,4,2',4'tetrahydroxychalcone (16), respectively, by spectroscopic analysis $\left({ }^{1} \mathrm{H}-\mathrm{NMR},{ }^{13} \mathrm{C}-\mathrm{NMR}, \mathrm{UV}, \mathrm{IR}\right.$ and MS) and comparisons with literature data. All these compounds have been isolated from this plant for the first time.

Compound 8 was obtained as a brown powder with optical rotation $[\alpha]_{\mathrm{D}}^{25}:-16.9^{\circ}(\mathrm{MeOH}, c=0.1)$, and its molecular formula was determined to be $\mathrm{C}_{10} \mathrm{H}_{12} \mathrm{O}_{3}$, as indicated by the $[\mathrm{M}+\mathrm{Na}]^{+}$ion at $\mathrm{m} / \mathrm{z}$ 203.0679 (calcd. for $203.0687[\mathrm{M}+\mathrm{Na}]^{+}$) in the HRESIMS spectrum. In the IR spectrum, absorption bands attributable to acetylene $\left(2341 \mathrm{~cm}^{-1}, 2360 \mathrm{~cm}^{-1}\right)$, hydroxyl $\left(3182 \mathrm{~cm}^{-1}\right)$ and ethylene $\left(1627 \mathrm{~cm}^{-1}\right)$ groups were observed. The UV spectrum of $\mathbf{8}$ was typical for an ene-diyne chromophore $\left(\lambda_{\max }=228\right.$, 240, 252, 266, $282 \mathrm{~nm}$ ) [18]. The ${ }^{13} \mathrm{C}-\mathrm{NMR}$ (Table 1) and DEPT spectra of $\mathbf{8}$ present 10 carbon signals, including three methylene groups at $\delta 41.4,59.1$ and 62.6, one methine group at $\delta 60.4$, two olefinic carbons at $\delta 108.6$ and 148.2, and four quaternary carbons at $\delta 69.5,74.1,77.5$ and 84.2 which were confirmed to be ethynyl carbons. Extensive analysis of the ${ }^{1} \mathrm{H}-\mathrm{NMR}$ spectrum, together with ${ }^{1} \mathrm{H}-$ 
${ }^{1} \mathrm{H}$ COSY and HMQC spectra, presented a methylene proton at $\delta 4.13(2 \mathrm{H}, \mathrm{dd}, J=2.1,4.7 \mathrm{~Hz}, \mathrm{H}-10)$, coupled with two $E$-configured olefinic protons at $\delta 6.39(1 \mathrm{H}, \mathrm{td}, J=4.7,15.9 \mathrm{~Hz}, \mathrm{H}-8)$ and $\delta 5.79$ $(1 \mathrm{H}, \mathrm{dtd}, J=0.7,2.1,15.9 \mathrm{~Hz}, \mathrm{H}-9)$ indicating a methylene allyl moiety, two methylene protons at $\delta$ $3.69(2 \mathrm{H}, \mathrm{m}, \mathrm{H}-1)$ and $\delta 1.88(2 \mathrm{H}, \mathrm{m}, J=6.8 \mathrm{~Hz}, \mathrm{H}-2)$, one methine proton at $\delta 4.57(1 \mathrm{H}, \mathrm{t}, J=6.8 \mathrm{~Hz}$, $\mathrm{H}-3)$. In the COSY spectrum, the correlations between $\delta 3.69(\mathrm{H}-1)$ and $\delta 1.88(\mathrm{H}-2), \delta 1.88(\mathrm{H}-2)$ and $\delta 4.57$ (H-3) suggested the presence of a $\mathrm{CH}_{2} \mathrm{CH}_{2} \mathrm{CH}$ moiety. In the $\mathrm{HMBC}$ spectrum, heteronuclear multiple-bond connectivity between the following: $\delta_{\mathrm{H}} 5.79(\mathrm{H}-9) / \delta_{\mathrm{C}} 77.5, \delta_{\mathrm{H}} 6.39(\mathrm{H}-8) / \delta_{\mathrm{C}} 77.5$ and $\delta_{\mathrm{C}}$ $74.1, \delta_{\mathrm{H}} 4.13(\mathrm{H}-10) / \delta_{\mathrm{C}} 77.5(\mathrm{C}-7)$ could be observed; furthermore, the intensity of correlations between $\delta_{\mathrm{H}} 6.39 / \delta_{\mathrm{C}} 77.5$ was weaker than that between $\delta_{\mathrm{H}} 6.39 / \delta_{\mathrm{C}} 74.1$, suggesting that $\delta_{\mathrm{C}} 74.1$ and $\delta_{\mathrm{C}}$ 77.5 form a alkynyl group and $\delta_{\mathrm{C}} 77.5$ directly connected with $\delta_{\mathrm{C}}(148.2)$ of the $\mathrm{CH}=\mathrm{CHCH}_{2}$ moiety, while $\delta_{\mathrm{C}} 69.5$ and $\delta_{\mathrm{C}} 84.7$ form another alkynyl. The peak at $\delta_{\mathrm{H}} 4.57$ (H-3) correlates simultaneously with $\delta_{\mathrm{C}} 84.7,69.5,77.5$ and 74.1, and together with $\delta_{\mathrm{H}} 5.37$ (H-9) presents a correlation with $\delta_{\mathrm{C}} 69.5$, suggesting two adjacent alkynyls, and $\delta_{\mathrm{C}} 60.4$ of the $\mathrm{CH}_{2} \mathrm{CH}_{2} \mathrm{CH}$ moiety is connected to $\delta_{\mathrm{C}} 84.7$. Thus, based on the chemical shifts of protons and carbons, the planar structure of compound 8 was determined to be 8 - $(E)$-decene-4,6-diyne-1,3,10-triol. All ${ }^{1} \mathrm{H}$ - and ${ }^{13} \mathrm{C}$-NMR signals as shown in Table 1 were assigned according to DEPT, HMQC, HMBC and ${ }^{1} \mathrm{H}-{ }^{1} \mathrm{H}$ COSY experiments. Figure 2 shows the key correlations presented in the ${ }^{1} \mathrm{H}-{ }^{1} \mathrm{H}$ COSY and HMBC spectra of 8.

Table $1 .{ }^{13} \mathrm{C}-\mathrm{NMR}\left(100 \mathrm{MHz}\right.$, in $\left.\mathrm{CD}_{3} \mathrm{OD}\right)$ and ${ }^{1} \mathrm{H}-\mathrm{NMR}(400 \mathrm{MHz})$ data of compound 8.

\begin{tabular}{cccc}
\hline $\begin{array}{c}\text { Positio } \\
\text { n }\end{array}$ & $\boldsymbol{\delta}_{\mathbf{C}}(\mathbf{p p m})$ & $\boldsymbol{\delta}_{\mathbf{H}}(\mathbf{p p m})$ & HMBC (H to C) \\
\hline 1 & 59.1 & $3.69(2 \mathrm{H}, \mathrm{m})$ & $\mathrm{C}-2,3$ \\
2 & 41.4 & $1.88(2 \mathrm{H}, \mathrm{m})$ & $\mathrm{C}-1,3,4$ \\
3 & 60.4 & $4.57(1 \mathrm{H}, \mathrm{t}, J=6.8 \mathrm{~Hz})$ & $\mathrm{C}-1,2,4,5,6,7$ \\
4 & 84.2 & & \\
5 & 69.5 & & \\
6 & 74.1 & & \\
7 & 77.5 & & $\mathrm{C}-6,7$ \\
8 & 148.2 & & $\mathrm{C}-5,6,8,9$ \\
9 & 108.6 & $5.79(1 \mathrm{H}, \mathrm{dtd}, J=0.7,2.1,15.9 \mathrm{~Hz})$ & $\mathrm{C}-7,8,9$ \\
\hline
\end{tabular}

Figure 2. The key $\mathrm{HMBC}$ and ${ }^{1} \mathrm{H}-{ }^{1} \mathrm{H}$ COSY correlations of compound $\mathbf{8}$.

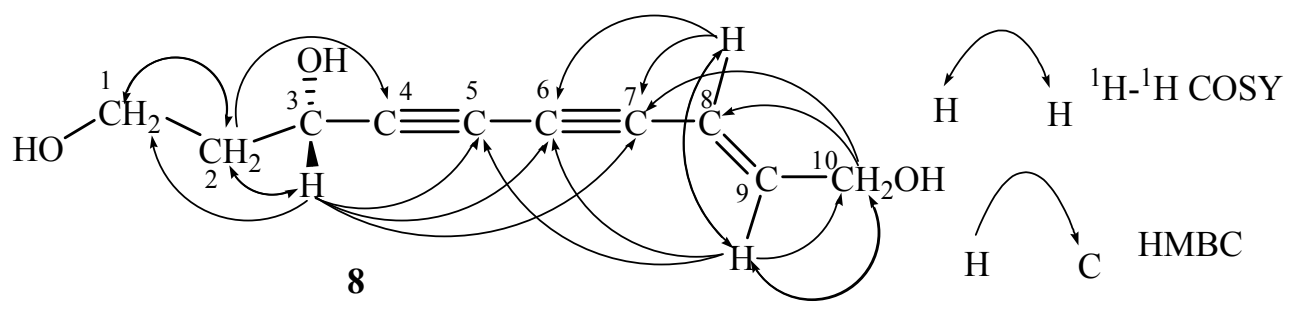


The stereochemistry at the chiral center (C-3) in compound $\mathbf{8}$ was determined using the modified Mosher method on the $R$-(+)- $\alpha$-methoxy- $\alpha$-trifluoromethylphenylacytyl (MTPA) and $S$-(-)-MTPA esters of 8. In the ${ }^{1} \mathrm{H}-\mathrm{NMR}$ spectrum of the $R-(+)$-MTPA ester, the $\mathrm{H}-1$ and $\mathrm{H}-2$ protons appeared upfield, suggesting an effect of the MTPA pheyl ring [19]. In contrast, H-8, H-9 and H-10 were downfield from the corresponding $S$-(-)-MTPA ester. The result was shown in Figure 3 . Thus, the absolute configuration at $\mathrm{C}-3$ of $\mathbf{8}$ was determined to be $R$.

Figure 3. $\delta=\delta_{S}-\delta_{R}$ values (ppm) obtained from the MTPA ester of 8 in $\mathrm{CDCl}_{3}$ at $25{ }^{\circ} \mathrm{C}$.

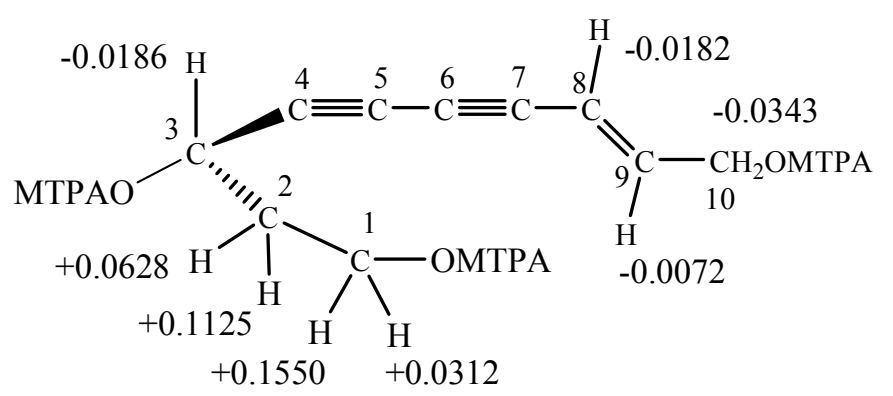

\section{Experimental}

\section{General}

UV spectra were measured in $\mathrm{MeOH}$ using a Shimadzu UV2401PC spectrophotometer (Shimadzu Co., Japan) and optical rotations were determined on a JASCO P-1020 polarimeter in MeOH. IR spectra were recorded on a Shimadau FTIR8400 spectrophotometer (Shimadzu Co., Japan) using KBr discs as stated. ESI-MS were obtained using a Bruker Esquire 2000 mass spectrometer (Bruker Co., Germany) and HR-ESI-MS were recorded on a Micromass Q-TOF mass spectrometer. NMR spectra were recorded on a Bruker AVANCE $400 \mathrm{NMR}$ spectrometer $\left({ }^{1} \mathrm{H}\right.$ at $400 \mathrm{MHz},{ }^{13} \mathrm{C}$ at $100 \mathrm{MHz}$, Bruker Co., Germany). Chemical shifts $(\delta)$ are shown in ppm relative to TMS as internal standard and coupling constants $(J)$ are given in Hz. The solvent used was DMSO- $\mathrm{d}_{6}$, unless otherwise stated. Preparative HPLC was carried out on a Shimadzu Pak equipped with a UV-Vis detector (Shimadzu Co., Japan) using a Shim-pack PREP-ODS column (5 $\mu, 10 \times 250 \mathrm{~mm}$, Shimadzu Co., Japan). Open column chromatography was carried out on D101 macroporous adsorption resin (Tianjin Nankai Daxue Chemical Plant, P. R. China), silica gel H60 (200-300 mesh, Qingdao Haiyang Chemical Group Co., P. R. China), Sephadex LH-20 (Amersham Pharmacia Biotech Co., UK) and ODS (60-80 $\mu \mathrm{m}$, Merck Co., Germany) as packing materials. Thin-layer chromatography was performed on silica gel H60 plates (Qingdao Haiyang Chemical Group Co., P.R. China) and RP-18 plate (Merck Co., Germany).

\section{Plant material}

The whole plant material of Bidens parviflora Willd. was collected in July 2003 in Liaoning province, P.R. China, and was identified by Prof. Weichun Wu (Department of Medical Plants, Shenyang Pharmaceutical University, Shenyang, P.R. China). A voucher specimen (99-DHS-953) was 
deposited in the herbarium of the Department of Natural Products Chemistry, Shenyang Pharmaceutical University, Shenyang, P. R. China.

\section{Extraction and Isolation}

The dried whole plant of B. parviflora Willd. $(6.0 \mathrm{~kg}$ ) was extracted three times with $60 \% \mathrm{EtOH}$ $(48 \mathrm{~L})$ under reflux. The resulting EtOH extract $(704 \mathrm{~g})$ was dissolved in water $(1.0 \mathrm{~L})$ and applied to a D101 macroporous adsorption resin column, then eluted with water, 30\% $\mathrm{EtOH}, 50 \% \mathrm{EtOH}$ and $95 \%$ $\mathrm{EtOH}$, respectively, to yield four fractions. The 30\% EtOH eluate (BPB, $72 \mathrm{~g}$ ) was subjected to silica gel column chromatography using a gradient solvent system $\left(\mathrm{CHCl}_{3}-\mathrm{MeOH}=100: 0 \rightarrow 98: 2 \rightarrow 95\right.$ : $5 \rightarrow 9: 1 \rightarrow 8: 2 \rightarrow 7: 3 \rightarrow 6: 4 \rightarrow 0: 1)$ to give twelve fractions BPB-1 to BPB-12. The BPB-3 fraction (5.45 g) was subjected to ODS column chromatography, eluted with gradient of increasing $\mathrm{MeOH}$ $\left(\mathrm{MeOH}-\mathrm{H}_{2} \mathrm{O}=20: 80 \rightarrow 40: 60 \rightarrow 60: 40 \rightarrow 90: 10\right)$, to give six fractions BPB-3a to BPB-3e; BPB-3c (940 mg) was purified by silica gel chromatography (gradient elution separation with a $\mathrm{CHCl}_{3}-\mathrm{MeOH}$ system: $95: 5 \rightarrow 9: 1 \rightarrow 85: 15 \rightarrow 8: 2$ ), followed by preparative HPLC (ODS, $\left.\mathrm{MeOH}-\mathrm{H}_{2} \mathrm{O}=48: 52\right)$ to yield compounds 1 (10 mg) and 2 (13 mg). BPB-3d (1.85 g) was subjected to Sephadex LH-20 column chromatography $\left(\mathrm{MeOH}-\mathrm{H}_{2} \mathrm{O}=50: 50\right)$, followed by preparative HPLC (ODS, $\left.\mathrm{MeOH}-\mathrm{H}_{2} \mathrm{O}=55: 45\right)$ to obtained $3(68 \mathrm{mg})$ and $4(25 \mathrm{mg})$. The BPB-6 fraction $(7.24 \mathrm{~g})$ was subjected to ODS column chromatography, eluted with an increasing gradient of $\mathrm{MeOH}\left(\mathrm{MeOH}-\mathrm{H}_{2} \mathrm{O}=10: 90 \rightarrow 30: 70 \rightarrow\right.$ $50: 50 \rightarrow 70: 30)$ to give six fractions BPB-6a to BPB-6e. BPB-6b $(2.40 \mathrm{~g})$ and BPB-6c $(2.02 \mathrm{~g})$ were subjected to Sephadex LH-20 column chromatography $\left(\mathrm{MeOH}-\mathrm{H}_{2} \mathrm{O}=1: 1\right)$, followed by ODS column chromatography and preparative HPLC (ODS, MeOH- $\left.\mathrm{H}_{2} \mathrm{O}=35: 65\right)$ to yield 5 (12 mg), 6 (45 mg), 7 (13 mg), 8 (30 mg) and 9 (22 mg).

The $60 \%$ EtOH eluate (BPC, $48 \mathrm{~g}$ ) was subjected to silica gel column chromatography using a gradient solvent system (hexane-acetone $=100: 0 \rightarrow 98: 2 \rightarrow 95: 5 \rightarrow 9: 1 \rightarrow 8: 2 \rightarrow 7: 3 \rightarrow 6: 4 \rightarrow 0: 1$ ) to give twelve fractions BPC-1 to BPC-12. Fraction BPC-1 (3.34 g) was subjected to silica gel column chromatography, eluted with hexane and ethyl acetate in increasing order of polarity, to give eight fractions BPC-1a to BPC-1h. BPC-1g (107 mg) was purified with Sephadex LH-20 column chromatography $\left(\mathrm{CHCl}_{3}-\mathrm{MeOH}=1: 1\right)$ to yield $10(23 \mathrm{mg})$. BPC-3 $(5.43 \mathrm{~g})$ and BPC-6 (3.06 g) were subjected to Sephadex LH-20 column chromatography $\left(\mathrm{CHCl}_{3}-\mathrm{MeOH}=1: 1\right)$ followed by silica gel column chromatography (gradient elution separation with $\mathrm{CHCl}_{3}$-acetone system) to obtain $\mathbf{1 1}(30 \mathrm{mg}$ ), 12 (24 mg), 13 (15 mg), 14 (20 mg), 15 (16 mg) and 16 (10 mg).

Acacetin 7-O-( $\alpha$-D-apio-furanosyl) $(1 \rightarrow 6)-\beta$-D-glucoside $(\mathbf{1})$ [20]: $\mathrm{C}_{27} \mathrm{H}_{30} \mathrm{O}_{14}$; yellow needle $(\mathrm{MeOH})$; mp 244-245 ${ }^{\circ} \mathrm{C}$; $[\alpha]_{\mathrm{D}} 23.0^{\circ}\left(\mathrm{MeOH}, c=0.23,24{ }^{\circ} \mathrm{C}\right.$ ); ESI-MS (positive) $\mathrm{m} / \mathrm{z} 579[\mathrm{M}+\mathrm{H}]^{+}$; UV $(\mathrm{MeOH}) \lambda_{\max } \mathrm{nm}(\log \varepsilon): 268$ (4.34), 325 (4.40); IR $v_{\max }(\mathrm{KBr}): 3424,2927,1652,1610,1500,1432$, 1303, 1257, 1174, 1058, $831 \mathrm{~cm}^{-1}$; ${ }^{1} \mathrm{H}-\mathrm{NMR} \delta: 8.06\left(2 \mathrm{H}, \mathrm{d}, J=9.1 \mathrm{~Hz}, \mathrm{H}-2^{\prime}, 6^{\prime}\right), 7.13(2 \mathrm{H}, \mathrm{d}, J=9.1$ Hz, H-3', 5'), 6.95 (1H, s, H-3), 6.46 (1H, d, $J=2.3 \mathrm{~Hz}, \mathrm{H}-6), 6.82(1 \mathrm{H}, \mathrm{d}, J=2.3 \mathrm{~Hz}, \mathrm{H}-8)$, , 3.87 (3H, $\left.\mathrm{s}, 4^{\prime}-\mathrm{OCH}_{3}\right), 5.07(1 \mathrm{H}, \mathrm{d}, J=7.5 \mathrm{~Hz}, \mathrm{glc}-\mathrm{H}-1), 4.82\left(1 \mathrm{H}, \mathrm{d}, J=3.2 \mathrm{~Hz}\right.$, api-H-1); ${ }^{13} \mathrm{C}-\mathrm{NMR} \delta: 163.8(\mathrm{~s}$, C-2), 103.8 (d, C-3), 182.0 (s, C-4), 161.0 (s, C-5), 99.6 (d, C-6), 162.9 (s, C-7), 94.8 (d, C-8), 156.9 (s, C-9), 105.4 (s, C-10), 122.7 (s, C-1'), 114.6 (d, C-2', 6'), 128.4 (d, C-3', 5'), 162.4 (s, C-4'), 55.6 (q, 4'$\mathrm{OCH}_{3}$ ), 99.8 (d, glc-C-1), 72.9 (d, glc-C-2), 76.2 (d, glc-C-3), 69.6 (d, glc-C-4), 75.5 (d, glc-C-5), 67.3 
(t, glc-C-6), 109.1 (d, api-C-1), 75.9 (d, api-C-2), 78.7 (s, api-C-3), 73.3 (t, api-C-4), 63.3 (t, api-C-5). Resonance assignments were based on ${ }^{1} \mathrm{H}-{ }^{1} \mathrm{H}$ COSY, HMQC and HMBC spectra.

Luteolin 7-O- $\beta$-D-neohesperidoside (2) [21, 22]: $\mathrm{C}_{28} \mathrm{H}_{32} \mathrm{O}_{15}$; yellow needles; mp 249-251 ${ }^{\circ} \mathrm{C}$; ESI-MS (positive) $m / z 609[\mathrm{M}+\mathrm{H}]^{+} ;{ }^{1} \mathrm{H}-\mathrm{NMR} \delta: 7.44\left(1 \mathrm{H}, \mathrm{dd}, J=8.3,2.1 \mathrm{~Hz}, \mathrm{H}-6{ }^{\prime}\right), 7.40(1 \mathrm{H}, \mathrm{d}, J=2.1 \mathrm{~Hz}$, H-2'), 6.90 (1H, $\left.J=8.3 \mathrm{~Hz}, \mathrm{H}-5^{\prime}\right), 6.75(1 \mathrm{H}, \mathrm{s}, \mathrm{H}-3), 6.74(1 \mathrm{H}, \mathrm{d}, J=2.1 \mathrm{~Hz}, \mathrm{H}-8), 6.38$ (1H, d, $J=2.1$ Hz, H-6), $5.25(1 \mathrm{H}, \mathrm{d}, J=7.3 \mathrm{~Hz}$, glc-H-1), $5.14(1 \mathrm{H}, \mathrm{d}, J=1.2 \mathrm{~Hz}$, rha-H-1), 1.20 (3H, d, $J=6.2 \mathrm{~Hz}$, rha- $\left.\mathrm{CH}_{3}\right) ;{ }^{13} \mathrm{C}-\mathrm{NMR} \delta$ : 166.3 (s, C-2), 105.1 (d, C-3), 183.7 (s, C-4), 163.0 (s, C-5), 99.6 (d, C-6), 164.4 (s, C-7), 92.6 (d, C-8), 158.8 (s, C-9), 107.3 (s, C-10), 123.2 (s, C-1'), 115.4 (d, C-2'), 147.6 (s, C-3'), 151.8 (s, C-4'), 117.9 (d, C-5'), 121.0 (d, C-6'), 102.3 (d, glc-C-1), 101.2 (d, rha-C-1), 78.8 (d, glc-C-2), 79.0 (d, glc-C-3), 72.2 (d, glc-C-4), 78.2 (d, glc-C-5), 62.4 (d, glc-C-6), 72.4 (d, rha-C-2), 71.5 (d, rha-C-3), 73.7 (d, rha-C-4), 70.2 (d, rha-C-5), 19.9 (q, rha- $\mathrm{CH}_{3}$ ).

Quercetin 3-O- $\beta$-D-rutinoside (3) [23]: $\mathrm{C}_{27} \mathrm{H}_{30} \mathrm{O}_{16}$; yellow powder; $[\alpha]_{\mathrm{D}}^{26}-2.8^{\circ}(c=0.1, \mathrm{MeOH})$; ESI$\mathrm{MS}^{\mathrm{n}}$ (positive and negative) $\mathrm{m} / z 633[\mathrm{M}+\mathrm{Na}]^{+}, 487[\mathrm{M}+\mathrm{Na}-146]^{+}, 609[\mathrm{M}-\mathrm{H}]^{-}, 301[\mathrm{M}-\mathrm{H}-(146+162)]^{-}$; UV (MeOH) $\lambda_{\max } \mathrm{nm}(\log \varepsilon): 355$ (4.09), 256 (4.20); ${ }^{1} \mathrm{H}-\mathrm{NMR} \delta: 6.21$ (1H, d, $\left.J=2.2 \mathrm{~Hz}, \mathrm{H}-6\right), 6.40$ $(1 \mathrm{H}, \mathrm{d}, J=2.2 \mathrm{~Hz}, \mathrm{H}-8), 7.67\left(1 \mathrm{H}, \mathrm{d}, J=2.5 \mathrm{~Hz}, \mathrm{H}-2^{\prime}\right), 6.87\left(\mathrm{H}, \mathrm{d}, J=8.3 \mathrm{~Hz}, \mathrm{H}-5^{\prime}\right), 7.63(1 \mathrm{H}, \mathrm{dd}, J=$ 8.6, $2.2 \mathrm{~Hz}, \mathrm{H}-6$ '), $5.11(1 \mathrm{H}, \mathrm{d}, J=7.7 \mathrm{~Hz}, \mathrm{glc}-\mathrm{H}-1) 4.52(1 \mathrm{H}, \mathrm{d}, J=1.5 \mathrm{~Hz}$, rha-H-1), $1.12(3 \mathrm{H}, \mathrm{d}, J=$ 6.1 Hz, rha-H-6); ${ }^{13} \mathrm{C}-\mathrm{NMR} \delta: 159.4$ (C-2), 135.7 (s, C-3), 179.5 (s, C-4), 163.0 (s, C-5), 99.9 (d, C-6), 166.1 (s, C-7), 94.9 (d, C-8), 158.6 (s, C-9), 105.7 (s, C-10), 123.2 (s, C-1'), 116.1 (d, C-2'), 145.9 (s, C-3'), 149.9 (s, C-4'), 117.7 (d, C-5'), 123.6 (d, C-6'), 104.8 (d, glc-C-1), 75.8 (d, glc-C-2), 78.2 (d, glc-C-3), 71.4 (d, glc-C-4), 77.3 (d, glc-C-5), 68.6 (t, glc-C-6), 102.5 (d, rha-C-1), 72.3 (d, rha-C-2), 72.1 (d, rha-C-3), 74.0 (d, rha-C-4), 69.8 (d, rha-C-5), 17.9 (q, rha-C-6).

Luteolin 7-O- $\beta$-D-glucoside (4) [24]: $\mathrm{C}_{21} \mathrm{H}_{20} \mathrm{O}_{11}$; yellow needles; mp 257-259 ${ }^{\circ} \mathrm{C}$; ESI-MS (positive) $m / z 449[\mathrm{M}+\mathrm{H}]^{+} ;{ }^{1} \mathrm{H}-\mathrm{NMR}\left(\mathrm{DMSO}-d_{6}\right) \delta: 13.0(1 \mathrm{H}, \mathrm{s}, \mathrm{br}, 5-\mathrm{OH}), 6.74(1 \mathrm{H}, \mathrm{s}, \mathrm{H}-3), 6.44(1 \mathrm{H}, \mathrm{d}, J=$ $2.1 \mathrm{~Hz}, \mathrm{H}-6), 6.78(1 \mathrm{H}, \mathrm{d}, J=2.1 \mathrm{~Hz}, \mathrm{H}-8), 7.42\left(1 \mathrm{H}, \mathrm{d}, J=2.2 \mathrm{~Hz}, \mathrm{H}-2^{\prime}\right), 6.90(1 \mathrm{H}, \mathrm{d}, J=8.2 \mathrm{~Hz}, \mathrm{H}-$ $\left.5^{\prime}\right), 7.44\left(1 \mathrm{H}, \mathrm{dd}, J=8.2,2.2 \mathrm{~Hz}, \mathrm{H}-6\right.$ '), $5.08\left(1 \mathrm{H}, \mathrm{d}, J=7.3 \mathrm{~Hz}\right.$, glc-H-1); ${ }^{13} \mathrm{C}-\mathrm{NMR}$ (DMSO- $\left.d_{6}\right) \delta$ : 163.7 (s, C-2), 102.4 (d, C-3), 181.1 (s, C-4), 160.3 (s, C-5), 98.8 (d, C-6), 162.1 (s, C-7), 93.9 (d, C-8), 156.2 (s, C-9), 104.5 (s, C-10), 120.6 (s, C-1'), 112.8 (d, C-2'), 145.0 (s, C-3'), 149.1 (s, C-4'), 115.2 (d, H-5'), 118.4 (d, H-6'), 99.1 (d, glc-C-1), 72.3 (d, glc-C-2), 76.4 (d, glc-C-3), 68.8 (d, glc-C-4), 75.6 (d, glc-C-5), 59.8 (t, glc-C-6).

Acacetin 7-O- $\beta$-D-glucoside (5) [25]: $\mathrm{C}_{22} \mathrm{H}_{22} \mathrm{O}_{10}$; yellow powder; ESI-MS (positive) $\mathrm{m} / z 447$ [M+H] ; ${ }^{1} \mathrm{H}-\mathrm{NMR} \delta$ : $12.92(1 \mathrm{H}, \mathrm{s}, 5-\mathrm{OH}), 6.96(1 \mathrm{H}, \mathrm{s}, \mathrm{H}-3), 6.46(1 \mathrm{H}, \mathrm{d}, J=2.2 \mathrm{~Hz}, \mathrm{H}-6), 6.86(1 \mathrm{H}, \mathrm{d}, J=2.2$ Hz, H-8), 8.07 (2H, d, $\left.J=9.0 \mathrm{~Hz}, \mathrm{H}-2^{\prime}, \mathrm{H}-6^{\prime}\right), 7.14\left(2 \mathrm{H}, \mathrm{d}, J=9.0 \mathrm{~Hz}, \mathrm{H}-3^{\prime}, \mathrm{H}-5^{\prime}\right), 5.07$ (1H, d, $J=7.7$ $\mathrm{Hz}$, glc-H-1), 3.89 (3H, s, 4'-OCH ${ }_{3}$ ) ${ }^{13} \mathrm{C}-\mathrm{NMR} \delta: 163.8$ (s, C-2), 103.7 (d, C-3) , 181.7 (s, C-4), 161.0 (s, C-5), 99.5 (d, C-6), 162.9 (s, C-7), 94.8 (d, C-8), 156.8 (s, C-9), 105.3 (s, C-10), 122.6 (s, C-1'), 128.4 (d, C-2'), 114.6 (s, C-3'), 162.4 (s, C-4'), 114.6 (d, H-5'), 128.4 (d, H-6'), 99.8 (d, glc-C-1), 72.9 (d, glc-C-2), 77.1 (d, glc-C-3), 69.5 (d, glc-C-4), 76.3 (d, glc-C-5), 60.5 (t, glc-C-6), 55.5 (q, 4'-OCH . $^{\prime}$

Quercitrin (6) [26]: $\mathrm{C}_{21} \mathrm{H}_{20} \mathrm{O}_{11}$; yellow powder; ESI-MS (positive) $m / z 449[\mathrm{M}+\mathrm{H}]{ }^{+} ;{ }^{1} \mathrm{H}-\mathrm{NMR} \delta: 12.65$ $(1 \mathrm{H}, \mathrm{s}, 5-\mathrm{OH}), 6.86\left(1 \mathrm{H}, \mathrm{d}, J=8.3 \mathrm{~Hz}, \mathrm{H}-5^{\prime}\right), 6.39(1 \mathrm{H}, \mathrm{d}, J=2.2 \mathrm{~Hz}, \mathrm{H}-8), 7.30(1 \mathrm{H}, \mathrm{d}, J=2.1 \mathrm{~Hz}, \mathrm{H}-$ 
$\left.2^{\prime}\right), 7.25\left(1 \mathrm{H}, \mathrm{dd}, J=2.1,8.3 \mathrm{~Hz}, \mathrm{H}-6^{\prime}\right), 6.20(1 \mathrm{H}, \mathrm{d}, J=2.2 \mathrm{~Hz}, \mathrm{H}-6), 5.25(1 \mathrm{H}, \mathrm{d}, J=1.4 \mathrm{~Hz}$, rha-H1), $3.97\left(1 \mathrm{H}, \mathrm{t}, J=1,4 \mathrm{~Hz}\right.$, rha-H-2), $0.81(3 \mathrm{H}, \mathrm{d}, J=6.0 \mathrm{~Hz}$, rha-CH$) ;{ }^{13} \mathrm{C}-\mathrm{NMR} \delta: 157.2(\mathrm{~s}, \mathrm{C}-2)$, 134.2 (s, C-3), 177.7 (s, C-4), 161.3 (s, C-5), 98.7 (d, C-6), 164.2 (s, C-7), 93.6 (d, C-8), 156.4 (s, C-9), 104.0 (s, C-10), 120.7 (s, C-1'), 115,4 (d, C-2'), 145.2 (s, C-3'), 148.4 (s, C-4'), 115.6 (d, C-5'), 121.1 (d, C-6'), 101.8 (d, rha-C-1), 70.5 (d, rha-C-2), 70.3 (d, rha-C-3), 71.2 (d, rha-C-4), 70.0 (d, rha-C-5), $17.4\left(\mathrm{q}\right.$, rha- $\left.\mathrm{CH}_{3}\right)$.

4-Methoxy-3,2',3'-trihydroxychalcone 4'-O- $\beta$-D-glucoside (7) [27, 28]: $\mathrm{C}_{22} \mathrm{H}_{24} \mathrm{O}_{11}$; yellow powder; ESI-MS (positive) $m / z 465[\mathrm{M}+\mathrm{H}]^{+}, 302[\mathrm{M}-162]^{+}$; UV (MeOH) $\lambda_{\max } \mathrm{nm}$ (loge): 229 (3.65), 312 (3.22); IR $v_{\max }(\mathrm{KBr}) \mathrm{cm}^{-1}: 3367,2925,1637,1567,1511,1448,1367,1272,1087 ;{ }^{1} \mathrm{H}-\mathrm{NMR}\left(\mathrm{CD}_{3} \mathrm{OD}\right) \delta: 7.79$ $(1 \mathrm{H}, \mathrm{d}, J=15.4 \mathrm{~Hz}, \beta-\mathrm{H}), 7.62(1 \mathrm{H}, \mathrm{d}, J=15.4 \mathrm{~Hz}, \alpha-\mathrm{H}), 7.25(1 \mathrm{H}, \mathrm{d}, J=2.2 \mathrm{~Hz}, \mathrm{H}-2), 7.00(1 \mathrm{H}, \mathrm{d}, J$ $=8.3 \mathrm{~Hz}, \mathrm{H}-5), 7.22(1 \mathrm{H}, \mathrm{dd}, J=8.3,2.2 \mathrm{~Hz}, \mathrm{H}-6), 7.65\left(1 \mathrm{H}, \mathrm{d}, J=9.2 \mathrm{~Hz}, \mathrm{H}-6^{\prime}\right), 6.86(1 \mathrm{H}, \mathrm{d}, J=9.2$ $\left.\mathrm{Hz}, \mathrm{H}-5^{\prime}\right), 3.91\left(3 \mathrm{H}, \mathrm{s}, 4-\mathrm{OCH}_{3}\right), 4.98\left(\mathrm{H}, \mathrm{d}, J=7.3 \mathrm{~Hz}\right.$, glc-H-1); ${ }^{13} \mathrm{C}-\mathrm{NMR}\left(\mathrm{CD}_{3} \mathrm{OD}\right) \delta: 119.3(\mathrm{~d}, \mathrm{C}-\alpha)$, 146.5 (d, C- $\beta$ ), 194.6 (s, C=O), 129.4 (s, C-1), 115.3 (d, C-2), 152.0 (s, C-3), 148.1 (s, C-4), 119.3 (d, C-5), 123.8 (d, C-6), 117.4 (s, C-1'), 146.5 (s, C-2'), 143.3 (s, C-3'), 165.1 (s, C-4'), 108.2 (d, H-5'), 122.7 (d, H-6'), 102.7 (d, glc-C-1), 74.8 (d, glc-C-2), 78.5 (d, glc-C-3), 71.3 (d, glc-C-4), 77.6 (d, glcC-5), 62.5 (t, glc-C-6), 56.5 (q, 4'- $\left.\mathrm{OCH}_{3}\right)$.

Quercetin 3-O- $\beta$-D-glucoside (9) [29]: $\mathrm{C}_{21} \mathrm{H}_{20} \mathrm{O}_{12}$; yellow powder; ESI-MS (positive and negative) $\mathrm{m} / \mathrm{z}$ $487[\mathrm{M}+\mathrm{Na}]^{+}, 325[\mathrm{M}+\mathrm{Na}-162]^{+}, 463[\mathrm{M}-\mathrm{H}]^{-}, 301[\mathrm{M}-\mathrm{H}-162]^{-} ; \mathrm{UV}(\mathrm{MeOH}) \lambda_{\max } \mathrm{nm}(\log \varepsilon): 356$ (4.05), 297 (3.83), 256 (4.13); ${ }^{1} \mathrm{H}-\mathrm{NMR} \delta$ : $12.62(1 \mathrm{H}, \mathrm{s}, 5-\mathrm{OH}), 10.85$ (1H, s, br, 7-OH), $9.72(1 \mathrm{H}, \mathrm{s}$, br, 4'-OH), $9.17\left(1 \mathrm{H}, \mathrm{s}, \mathrm{br}, 3^{\prime}-\mathrm{OH}\right), 6.20(1 \mathrm{H}, \mathrm{d}, J=2.0 \mathrm{~Hz}, \mathrm{H}-6), 6.41(1 \mathrm{H}, \mathrm{d}, J=2.0 \mathrm{~Hz}, \mathrm{H}-8), 7.53$ $\left(1 \mathrm{H}, \mathrm{d}, J=1.9 \mathrm{~Hz}, \mathrm{H}-2^{\prime}\right), 6.82\left(1 \mathrm{H}, \mathrm{d}, J=8.3 \mathrm{~Hz}, \mathrm{H}-5^{\prime}\right), 7.66\left(1 \mathrm{H}, \mathrm{dd}, J=8.3,1.9 \mathrm{~Hz}, \mathrm{H}-6^{\prime}\right), 5.37(1 \mathrm{H}$, $\mathrm{d}, J=7.6 \mathrm{~Hz}$, glc-H-1); ${ }^{13} \mathrm{C}-\mathrm{NMR} \delta: 156.2$ (s, C-2), 133.4 (s, C-3), 177.4 (s, C-4), 161.2 (s, C-5), 98.6 (d, C-6), 164.1 (s, C-7), 93.4 (d, C-8), 156.2 (s, C-9), 103.9 (s, C-10), 121.0 (s, C-1'), 115.1 (d, C-2'), 144.8 (s, C-3'), 148.4 (s, C-4'), 115.9 (d, C-5'), 121.9 (d, C-6'), 101.8 (d, glc-C-1), 74.0 (d, glc-C-2), 77.5 (d, glc-C-3), 69.8 (d, glc-C-4), 76.4 (d, glc-C-5), 60.8 (t, glc-C-6).

Taxifolin (10) [30]: $\mathrm{C}_{15} \mathrm{H}_{12} \mathrm{O}_{7}$; white powder; ESI-MS (positive) $m / z 327[\mathrm{M}+\mathrm{Na}]^{+} ;{ }^{1} \mathrm{H}-\mathrm{NMR} \delta$ : 11.87 $(1 \mathrm{H}, \mathrm{s}, 5-\mathrm{OH}), 10.79(1 \mathrm{H}, \mathrm{s}, 7-\mathrm{OH}), 8.99\left(1 \mathrm{H}, \mathrm{s}, 4^{\prime}-\mathrm{OH}\right), 8.94\left(1 \mathrm{H}, \mathrm{s}, 3^{\prime}-\mathrm{OH}\right), 4.96(1 \mathrm{H}, \mathrm{d}, J=11.2 \mathrm{~Hz}$, H-2), $4.48(1 \mathrm{H}, \mathrm{d}, J=11.2 \mathrm{~Hz}, \mathrm{H}-3), 5.84(1 \mathrm{H}, \mathrm{d}, J=2.1 \mathrm{~Hz}, \mathrm{H}-6), 5.89(1 \mathrm{H}, \mathrm{d}, J=2.1 \mathrm{~Hz}, \mathrm{H}-8), 6.85$ $\left(1 \mathrm{H}, \mathrm{s}, \mathrm{br}, \mathrm{H}-2^{\prime}\right), 6.71\left(1 \mathrm{H}, \mathrm{d}, J=8.0 \mathrm{~Hz}, \mathrm{H}-5^{\prime}\right), 6.73\left(1 \mathrm{H}, \mathrm{d}, J=8.0 \mathrm{~Hz}, \mathrm{H}-6^{\prime}\right) ;{ }^{13} \mathrm{C}-\mathrm{NMR} \delta: 83.5(\mathrm{~d}$, C-2), 72.0 (d, C-3), 198.2 (s, C-4), 163.8 (s, C-5), 96.4 (d, C-6), 167.2 (s, C-7), 95.4 (d, C-8), 163.0 (s, C-9), 101.0 (s, C-10), 128.5 (s, C-1'), 115.6 (d, C-2'), 145.4 (s, C-3'), 146.2 (s, C-4'), 115.8 (d, C-5'), $119.8\left(\mathrm{~d}, \mathrm{C}-6^{\prime}\right)$.

2'-Hydroxy-3,4,4',6'-tetramethoxychalcone (11) [31]: $\mathrm{C}_{19} \mathrm{H}_{20} \mathrm{O}_{6}$; yellow powder; ESI-MS (positive and negative) $m / z 345[\mathrm{M}+\mathrm{H}]^{+}, 343[\mathrm{M}-\mathrm{H}]^{-} ;{ }^{1} \mathrm{H}-\mathrm{NMR}$ (acetone- $\left.d_{6}\right) \delta: 7.91(1 \mathrm{H}, \mathrm{d}, J=15.4 \mathrm{~Hz}, \mathrm{H}-\alpha), 7.75$ $(1 \mathrm{H}, \mathrm{d}, J=15.4 \mathrm{~Hz}, \mathrm{H}-\beta), 7.33(1 \mathrm{H}, \mathrm{d}, J=2.0 \mathrm{~Hz}, \mathrm{H}-2), 7.03(1 \mathrm{H}, \mathrm{d}, J=8.2 \mathrm{~Hz}, \mathrm{H}-5), 7.30(1 \mathrm{H}, \mathrm{dd}, J$ $=8.2,2.0 \mathrm{~Hz}, \mathrm{H}-6), 6.13\left(1 \mathrm{H}, \mathrm{d}, J=2.4 \mathrm{~Hz}, \mathrm{H}-3^{\prime}\right), 6.09\left(1 \mathrm{H}, \mathrm{d}, J=2.4 \mathrm{~Hz}, \mathrm{H}-5^{\prime}\right), 4.01\left(3 \mathrm{H}, \mathrm{s}, 4^{\prime}-\right.$ $\left.\mathrm{OCH}_{3}\right), 3.91\left(3 \mathrm{H}, \mathrm{s}, 3-\mathrm{OCH}_{3}\right), 3.88\left(6 \mathrm{H}, 4,6^{\prime}-\mathrm{OCH}_{3}\right) ;{ }^{13} \mathrm{C}-\mathrm{NMR}$ (acetone- $\left.d_{6}\right) \delta: 193.4(\mathrm{~s}, \mathrm{C}=\mathrm{O}), 126.0$ (d, C- $\alpha$ ), 143.6 (d, C- $\beta$ ), 129.3 (s, C-1), 111.7 (d, C-2), 150.6 (s, C-3), 152.7 (s, C-4), 112.6 (d, C-5), 
123.7 (d, C-6), 106.9 (s, C-1'), 169.1 (s, C-2'), 91.8 (d, C-3'), 163.7 (s, C-4'), 94.7 (d, C-5'), 167.4 (s,

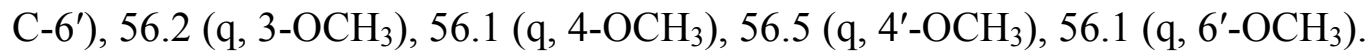

Kaempferol (12) [32]: $\mathrm{C}_{15} \mathrm{H}_{10} \mathrm{O}_{6}$; yellow powder; ESI-MS (negative) $\mathrm{m} / \mathrm{z} 285$ [M-H] ${ }^{-}$; UV (MeOH) $\lambda_{\max } \mathrm{nm}(\log \varepsilon): 254$ (4.15), 366 (4.02); ${ }^{1} \mathrm{H}-\mathrm{NMR}$ (acetone- $\left.d_{6}\right) \delta: 6.27(1 \mathrm{H}, \mathrm{d}, J=2.2 \mathrm{~Hz}, \mathrm{H}-6), 6.54$ $(1 \mathrm{H}, \mathrm{d}, J=2.2 \mathrm{~Hz}, \mathrm{H}-8), 8.14\left(2 \mathrm{H}, \mathrm{d}, J=8.0 \mathrm{~Hz}, \mathrm{H}-2^{\prime}, 6^{\prime}\right), 7.02\left(2 \mathrm{H}, \mathrm{d}, J=8.0 \mathrm{~Hz}, \mathrm{H}-3^{\prime}, 5^{\prime}\right) ;{ }^{13} \mathrm{C}-\mathrm{NMR}$ (acetone- $\left.d_{6}\right) 147.1(\mathrm{~s}, \mathrm{C}-2), 136.7$ (s, C-3), 176.6 (s, C-4), 162.4 (s, C-5), 99.2 (d, C-6), 165.0 (s, C-7), 94.5 (d, C-8), 157.8 (s, C-9), 104.2 (s, C-10), 123.4 (s, C-1'), 130.5 (d, C-2', 6'), 116.4 (d, C-3', 5'), $160.2\left(\mathrm{~s}, \mathrm{C}-4^{\prime}\right)$.

Luteolin (13) [25]: $\mathrm{C}_{15} \mathrm{H}_{10} \mathrm{O}_{6}$; yellow needles; mp 328-330 ${ }^{\circ} \mathrm{C}$; ESI-MS (positive) $\mathrm{m} / z 287$ [M+H] $]^{+} ;{ }^{1} \mathrm{H}-$ NMR $\delta$ : $6.74(1 \mathrm{H}, \mathrm{s}, \mathrm{H}-3), 6.44(1 \mathrm{H}, \mathrm{d}, J=1.9 \mathrm{~Hz}, \mathrm{H}-6), 6.79(1 \mathrm{H}, \mathrm{d}, J=1.9 \mathrm{~Hz}, \mathrm{H}-8), 7.42(1 \mathrm{H}, \mathrm{d}, J$ $\left.=2.2, \mathrm{H}-2^{\prime}\right), 6.90\left(1 \mathrm{H}, \mathrm{d}, J=8.2, \mathrm{H}-5^{\prime}\right), 7.45\left(1 \mathrm{H}, \mathrm{dd}, J=8.2,2.2, \mathrm{H}-6{ }^{\prime}\right) ;{ }^{13} \mathrm{C}-\mathrm{NMR} \delta: 164.5(\mathrm{~s}, \mathrm{C}-2)$, 103.1 (d, C-3), 181.9 (s, C-4), 161.1 (s, C-5), 99.1 (d, C-6), 163.9 (s, C-7), 94.2 (d, C-8), 156.9 (s, C-9), 104.3 (s, C-10), 121.3 (s, C-1'), 113.5 (d, C-2'), 145.8 (s, C-3'), 150.3 (s, C-4'), 115.9 (d, C-5'), 119.2 (d, C-6').

Quercetin (14) [25, 33]: $\mathrm{C}_{15} \mathrm{H}_{10} \mathrm{O}_{7}$; yellow needles; mp 310-312 ${ }^{\circ} \mathrm{C}$; ESI-MS (positive and negative) $m / z 303[\mathrm{M}+\mathrm{H}]^{+}, 301[\mathrm{M}-\mathrm{H}]^{-} ; \mathrm{UV}(\mathrm{MeOH}) \lambda_{\max } \mathrm{nm}(\log \varepsilon): 255$ (4.26), 370 (4.20); ${ }^{1} \mathrm{H}-\mathrm{NMR} \delta: 12.48$ $(1 \mathrm{H}, \mathrm{s}, 5-\mathrm{OH}), 10.75(1 \mathrm{H}, \mathrm{s}, \mathrm{br}, 3-\mathrm{OH}), 9.56(1 \mathrm{H}, \mathrm{s}, \mathrm{br}, 7-\mathrm{OH}), 9.32(2 \mathrm{H}, \mathrm{s}, \mathrm{br}, 2 \times-\mathrm{OH}), 6.19(1 \mathrm{H}, \mathrm{d}, J$ $=2.0 \mathrm{~Hz}, \mathrm{H}-6), 6.41(1 \mathrm{H}, \mathrm{d}, J=2.0 \mathrm{~Hz}, \mathrm{H}-8), 7.68\left(1 \mathrm{H}, \mathrm{d}, J=2.2 \mathrm{~Hz}, \mathrm{H}-2^{\prime}\right), 6.89(1 \mathrm{H}, \mathrm{d}, J=8.5 \mathrm{~Hz}$, H-5'), $7.54\left(1 \mathrm{H}, \mathrm{dd}, J=2.2,8.5 \mathrm{~Hz}, \mathrm{H}-6{ }^{\prime}\right) ;{ }^{13} \mathrm{C}-\mathrm{NMR} \delta$ : 147.6 (s, C-2), 135.6 (s, C-3), 175.8 (s, C-4), 160.6 (s, C-5), 98.1 (d, C-6), 163.8 (s, C-7), 93.3 (d, C-8), 156.1 (s, C-9), 103.0 (s, C-10), 121.9 (s, C1'), $115.0\left(\mathrm{~d}, \mathrm{C}-2^{\prime}\right), 145.0$ (s, C-3'), 146.7 (s, C-4'), 115.5 (d, C-5'), 120.0 (d, C-6').

Sulfuretin (15) [34]: $\mathrm{C}_{15} \mathrm{H}_{10} \mathrm{O}_{5}$; yellow powder; ESI-MS (positive and negative) $\mathrm{m} / z 271[\mathrm{M}+\mathrm{H}]^{+}$, 269.0 [M-H] ; ${ }^{1} \mathrm{H}-\mathrm{NMR} \delta: 6.84(1 \mathrm{H}, \mathrm{d}, J=8.3 \mathrm{~Hz}, \mathrm{H}-4), 6.70(1 \mathrm{H}, \mathrm{dd}, J=8.3,2.0 \mathrm{~Hz}, \mathrm{H}-5), 6.74(1 \mathrm{H}$, $\mathrm{d}, J=2.0 \mathrm{~Hz}, \mathrm{H}-7), 6.63(1 \mathrm{H}, \mathrm{s}, \mathrm{H}-10), 7.45\left(1 \mathrm{H}, \mathrm{d}, J=1.2 \mathrm{~Hz}, \mathrm{H}-2^{\prime}\right), 7.59\left(1 \mathrm{H}, \mathrm{d}, J=8.3 \mathrm{~Hz}, \mathrm{H}-5^{\prime}\right)$, $7.24\left(1 \mathrm{H}, \mathrm{dd}, J=8.3,1.2 \mathrm{~Hz}, \mathrm{H}-6^{\prime}\right) ;{ }^{13} \mathrm{C}-\mathrm{NMR} \delta: 145.6$ (s, C-2), 181.2 (s, C-3), 125.8 (d, C-4), 113.0 (d, C-5), 166.3 (s, C-6), 98.4 (d, C-7), 167.5 (s, C-8), 113.2 (s, C-9), 111.9 (d, C-10), 123.4 (s, C-1'), 118.0 (d, C-2'), 145.7 (s, C-3'), 148.0 (s, C-4'), 116.1 (d, C-5'), 124.6 (d, C-6').

3,4,2', 4'-Tetrahydroxychalcone (16) [35, 36]: $\mathrm{C}_{15} \mathrm{H}_{12} \mathrm{O}_{4}$; yellowish oily substance; ESI-MS (positive and negative) $m / z 287[\mathrm{M}+\mathrm{H}]^{+}, 285[\mathrm{M}-\mathrm{H}]^{-}$; UV (MeOH) $\lambda_{\max } \mathrm{nm}(\log \varepsilon): 218$ (3.50), 250 (2.91), 289 (0.54); IR $v_{\max }(\mathrm{KBr}) \mathrm{cm}^{-1}: 2554,1693,1597,1570,1504,1420,1377,1327 ;{ }^{1} \mathrm{H}-\mathrm{NMR}\left(\mathrm{CD}_{3} \mathrm{OD}\right) \delta$ : $6.70(1 \mathrm{H}, \mathrm{d}, J=15.3 \mathrm{~Hz}, \mathrm{H}-\alpha), 6.90(1 \mathrm{H}, \mathrm{d}, J=15.3 \mathrm{~Hz}, \mathrm{H}-\beta), 6.37(1 \mathrm{H}, \mathrm{s}, \mathrm{H}-2), 6.01(1 \mathrm{H}, \mathrm{d}, J=8.0$ Hz, H-5), $6.28(1 \mathrm{H}, \mathrm{d}, J=8.0 \mathrm{~Hz}, \mathrm{H}-6), 5.48\left(1 \mathrm{H}, \mathrm{d}, J=2.0 \mathrm{~Hz}, \mathrm{H}-3^{\prime}\right), 5.67(1 \mathrm{H}, \mathrm{dd}, J=8.9,2.0 \mathrm{~Hz}$, H-5') $7.11\left(1 \mathrm{H}, \mathrm{d}, J=8.9 \mathrm{~Hz}, \mathrm{H}-6^{\prime}\right) ;{ }^{13} \mathrm{C}-\mathrm{NMR}\left(\mathrm{CD}_{3} \mathrm{OD}\right) \delta$ : $193.5(\mathrm{~s}, \mathrm{C}=\mathrm{O}), 146.0(\mathrm{~d}, \mathrm{C}-\alpha), 118.3(\mathrm{~d}$, C- $\beta$ ), 128.4 (s, C-1), 115.8 (d, C-2), 146.8 (s, C-3), 149,8 (s, C-4), 116.6 (d, C-5), 123.6 (d, C-6), 114.7 (s, C-1'), $167.4\left(\mathrm{~s}, \mathrm{C}-2^{\prime}\right), 103.8$ (d, C-3') 166.2 (s, C-4'), 109.1 (d, C-5'), $133.2\left(\mathrm{~d}, \mathrm{C}-6^{\prime}\right)$. 


\section{Acknowledgements}

This work was supported by a grant from the National Natural Science Foundation of Shenzhen Bureau of Science Technology \& Information. The authors would like to thank Jing-hui Huang, and Ling Li (Key Lab for New Drugs Research of TCM, Research Institute of Tsinghua University in Shenzhen, P.R. China) for recording the MS and NMR data.

\section{References}

1. Jiangsu New Medical College. Dictionary of Chinese Crude Drugs; Shanghai Technological Publisher: Shanghai, P.R. China, 1986; pp. 1413, 1694, 2239.

2. Xia, G.; Zhang, J.; Chen, X.; Ma, L. The resource utilization of Bidens parviflora Willd. in Baoding district. Chin. Trad. Herb. Drugs 1985, 16, 37-40.

3. Ma, T. B.; Li, J. L.; Yuan, J. R. Isolation and identification of flavonoid glycosides from the leaf of smallflower beggarticks (Bidens parviflora). Chin. Trad. Herb. Drugs 1991, 22, 531-533.

4. Hoffman, B.; Hozel, J. Weitere acylierte Chalcone aus Bidens pilosa. Planta Med. 1988, 54, 450-451.

5. Hoffman, B.; Hozel, J. A methylated chalcone glucoside from Bidens pilosa. Phytochemistry 1988, 27, 3700-3703.

6. Tommasi, N. De; Piacente, S.; Pizza, C. Flavonol and Chalcone Ester Glycosides from Bidens andicola. J. Nat. Prod. 1998, 61, 973-977.

7. Sashida, Y.; Ogawa, K.; Kitada, M.; Karikome, H.; Mimaki, Y.; Shimomura, H. New aurone glucosides and phenylpropanoid glucosides from Bidens pilosa. Chem. Pharm. Bull. 1991, 39, 709-711.

8. Redl, K.; Davis, B.; Bauer, R. Chalcone glycosides from Bidens campylotheca. Phytochemistry 1993, 32, 218-220.

9. Bauer, R.; Redl, K.; Davis, B. Four polyacetylene glucosides from Bidens campyplotheca. Phytochemistry 1992, 31, 2035-2037.

10. Chen, A. H.; Lin, S. R.; Hong, C. H. Hua Hsueh 1975, 9, 42-48.

11. Wang, N. L.; Yao, X. S.; Ishii, R.; Kitanaka, S. Bioactive sucrose esters from Bidens parviflora willd. Phytochemistry 2003, 62, 741-746.

12. Wang, J.; Wang, N. L.; Yao, X. S.; Kitanaka, S. Structures and anti-histamine activities of phenolic acid derivatives from Bidens parviflora Willd. Chin. J. Med. Chem. 2006, 16, 168-171.

13. Wang, N. L.; Yao, X. S.; Ishii, R.; Kitanaka, S. Antiallergic agents from natural sources. 3. ${ }^{1)}$ Structures and inhibitory effects on nitric oxide production and histamine release of five novel polyacetylene glucosides from Bidens parviflora Willd. Chem. Pharm. Bull. 2001, 49, 938-942.

14. Wang, N. L.; Wang, J.; Yao, X. S.; Kitanaka, S. Two new monoterpene glycosides and a new (+)-jasmololone glucoside from Bidens parviflora Willd. J. Asian Nat. Prod. Res. 2007, 9, 449455.

15. Wang, N. L.; Wang, J.; Yao, X. S.; Kitanaka, S. Two neolignan glucosides and antihistamine release activities from Bidens parviflora Willd. Chem. Pharm. Bull. 2006, 54, 1190-1192. 
16. Wang, J.; Wang, N. L.; Yao, X. S.; Kitanaka, S. Phenolic glucosides from Bidens parviflora and their anti-histamine activities. Chin. Trad. Herb. Drugs 2007, 38, 647-649.

17. Wang, J.; Wang, N. L.; Yao, X. S.; Kitanaka, S. Caffeoylquinic acid derivatives from Bidens parviflora and their antihistamine release activites. Chin. Trad. Herb. Drugs 2006, 37, 966.

18. Bohlman, E.; Burkhardt, T.; Zdero, C. Naturally Occuring Acetylenes; Academic Press: London, UK, 1973.

19. Ohtani, I.; Kusumi, T.; Kashman, Y.; Kakisawa, H. High-field FT-IR application of Mosher's method-the absoluteconfiguration of marine terpenoids. J. Am. Chem. Soc. 1991, 113, 4092-4096.

20. Wanjala, C. W.; Majinda, R. T. Flavonoid glycosides from Crotalaria podocarpa. Phytochemistry 1999, 51, 705-707.

21. Kawashty, S. A.; El-Garf, I. A. The flavonoid chemosystematics of Egyptian Verbena species. Biochem. System. Ecol. 2000, 28, 919-921.

22. Zhang, Q. Y.; Wang, X. Y.; Ying, H. P.; Cheng, T. M.; Zhao, Y. Y. Studies on the Chemical Constituents of Carduus crispus L. China J. Chin. Mat. Med. 2001, 26, 837-839.

23. Wang, J. H.; Wang, Y. L.; Luo, F. C. Study on the chemical constituents from seeds Sophora japonica. Chin. Trad. Herb. Drugs 2001, 32, 471-473.

24. Harborne, J. B. The Flavonoids: Advances in Reseach since 1986; Chapman \& Hall: London, UK, 1994; pp. 450-451.

25. Jia, L. Y.; Sun, Q. S.; Huang, S. W. Isolation and identification of flavonoids from chrysanthemum moriflolium Ramat. Chin. J. Med. Chem. 2003, 13, 159-161.

26. Markham, K. R.; Ternai, B.; Stanley, R.; Geiger, K.; Mabry, T. J. Carbon ${ }^{-13}$ NMR studies of flavonoids-III naturally occurring flavonoid glycosides and their acylated derivatives. Tetrahedron 1978, 34, 1389-1397.

27. Nunziatina, D. T.; Cosimo, P. Flavonol and Chalcone Ester Glycosides from Bidens leucantha. J. Nat. Prod. 1997, 60, 270-273.

28. Nunziatina, D. T.; Sonia, P.; Cosimo, P. Flavonol and Chalcone Ester Glycosides from Bidens andicola. J. Nat. Prod. 1998, 61, 973-977.

29. Jia, Z. J.; Gong, N. C.; Du, M. Chemical constituents of Saussurea medusa Maxim. Chem. J. Chin. Univ. 1990, 11, 202-204.

30. HU, H. Y.; Yang, Y.; Yu, N. J.; Zhao, Y. M. Study on chemical constituents of bark of Paeonia suffruticos. China J. Chin. Mat. Med. 2006, 31, 1795-1797.

31. Phrutivorapongkul, A.; Lipipun, V.; Ruangrungsi, N.; Kirtikara, K.; Nishikkawa, K.; Maruyama, S.; Watanabe, T.; Ishikawa, T. Studies on the chemical constituents of stem bark of Millettia leucantha: Isolation of new chalcones with cytotoxic, anti-herpes simplex virus and antiinflammatory activities. Chem. Pharm. Bull. 2003, 51, 187-190.

32. Wei, Y. H.; Wu, X. A.; Zhang, C. Z.; Li, C.; Song, L. Studies on Chemical Constituents of Rheum glabricaule Sam.(II). Chin. Pharm. J. 2006, 41, 253-254.

33. Li, X.; Shi, R. B.; Liu, B.; Chen, Y. P. Study on chemical components from effective fraction of Qingnaoxuanqiao Formula (II). J. Beijing Univ. Trad. Chin. Med. 2006, 29, 545-550.

34. Venkateswarlu, S.; Panchagnula, G. K.; Subbaraju, G. V. Synthensis and antioxidative activity of 3', 4', 6, 7-tetrahydroxyaurone, a metabolite of Bidens frondosa. Biosci. Biotechnol. Biochem. 2004, 68, 2183-2185. 
35. Zhang, Y.; Shi, S. P.; Zhao, M. B.; Jiang, Y.; Tu, P. F. A novel chalcone from Coreopsis tinctoria Nutt. Biochem. System. Ecol. 2006, 34, 766-769.

36. Tian, G. 1.; Zhang, B.; Zhang, T. Y.; Yang, F. Q.; Yoichiro, I. Separation of flavonoids from the seeds of Vernonia anthelmintica Willd by high-speed counter-current chromatography. J. Chromatogr. A 2004, 1049, 219-222.

Sample Availability: Samples of the compounds 1, 3, 4, 6, 8, 9, 11-15 are available from the authors.

(C) 2008 by the authors; licensee Molecular Diversity Preservation International, Basel, Switzerland. This article is an open-access article distributed under the terms and conditions of the Creative Commons Attribution license (http://creativecommons.org/licenses/by/3.0/). 